\title{
Diferencias de género en el éxito al dejar de fumar: resultados a corto y largo plazo
}

\section{Gender differences in success at quitting smoking: Short- and long-term outcomes}

\author{
Adriana Marqueta*, Isabel Nerín*,**, Pilar Gargallo***, Asunción Beamonte*** \\ *Unidad de Tabaquismo. Universidad de Zaragoza, España; **Departamento de Medicina, Psiquiatría y Dermatología. \\ Facultad de Medicina, Universidad de Zaragoza, España; ***Departamento de Métodos Estadísticos, Facultad de Ciencias \\ Económicas y Empresariales, Universidad de Zaragoza, España.
}

\section{Resumen}

Los tratamientos para dejar de fumar son eficaces en hombres y mujeres. Sin embargo, las posibles diferencias encontradas en los resultados del tratamiento aún son objeto de controversia. Este estudio analiza si existen diferencias entre hombres y mujeres en el éxito al dejar de fumar a corto y largo plazo (> 1 año) con un programa de tratamiento que incluye la perspectiva de género. Se realizó una encuesta telefónica en fumadores atendidos en una unidad de tabaquismo. Los pacientes que completaron con éxito el tratamiento (3 meses), fueron encuestados telefónicamente para determinar su abstinencia a largo plazo; se validó la abstinencia mediante cooximetría (CO espirado $\leq 10 \mathrm{ppm}$ ) en los que se mantenían abstinentes. La probabilidad de permanecer abstinentes a largo plazo se calculó utilizando un análisis de supervivencia de Kaplan-Meier. La tasa de éxito del tratamiento fue de 41,3\% (538/1302), sin diferencias por sexo. El $89 \%$ (479/538) fue localizado por teléfono y el $47,6 \%$ $(256 / 479)$ se mantenía abstinente sin diferencias por sexo $(p=, 519)$; la abstinencia fue validada en 191 de 256 (53,9\% hombres y 46,1\% mujeres). En el análisis de supervivencia, la probabilidad de que los hombres y las mujeres mantuvieran la abstinencia a largo plazo no fue significativa. No hay diferencias por sexo en el resultado del tratamiento para dejar de fumar, que incluyan aspectos de género, a corto y largo plazo (> 1 año).

Palabras clave: Tabaquismo; cesación tabáquica; género y salud; mujeres; análisis de supervivencia.

\begin{abstract}
Smoking cessation treatments are effective in men and women. However, possible sex-related differences in the outcome of these treatments remain a controversial topic. This study evaluated whether there were differences between men and women in the success of smoking cessation treatment, including gender-tailored components, in the short and long term (> 1 year). A telephone survey was carried out between September 2008 and June 2009 in smokers attended in a Smoking Cessation Clinic. All patients who have successfully completed treatment (3 months) were surveyed by telephone to determine their long-term abstinence. Those who remained abstinent were requested to attend the Smoking Cessation Clinic for biochemical validation (expired $\mathrm{CO} \leq 10 \mathrm{ppm}$ ). The probability of remaining abstinent in the long-term was calculated using a KaplanMeier survival analysis. The treatment success rate at 3-months was $41.3 \%$ (538/1302) with no differences by sex $89 \%$ (479/538) among those located in the telephonic follow-up study and $47.6 \%(256 / 479)$ were abstinent without differences by sex $(p=.519)$; abstinence was validated with CO less than 10 ppm in 191 of the $256(53.9 \%$ men and $46.1 \%$ women). In the survival analysis, the probability of men and women remaining abstinent in the long-term was not significant. There are no differences by sex in the outcome of smoking cessation treatment that included gender-tailored components in the short and long term (> 1 year).

Keywords: Smoking; smoking cessation; gender and health; women; survival analysis.
\end{abstract}


$\mathrm{E}$ 1 tabaquismo es el problema de salud prioritario de los países desarrollados y un problema emergente en los países en vías de desarrollo (López, Mathers, Ezzati, Jamison, y Murray, 2006). A nivel global, la prevalencia de fumadores es más alta en hombres que en mujeres, aunque está aumentando entre mujeres jóvenes (Amos, Greaves, Nichter, y Bloch, 2012). Como resultado de estas diferencias en la prevalencia de tabaquismo por sexo, hasta la fecha la mortalidad asociada al tabaquismo ha sido más elevada entre los hombres. No obstante, en algunos países desarrollados, el aumento del hábito de fumar entre las mujeres también ha provocado un aumento en la mortalidad asociada entre las mujeres, en comparación con años anteriores. Por ello, en muchos países el consumo de tabaco entre las mujeres ya se ha convertido en una preocupación importante de los sistemas de salud públicos (Croghan et al., 2009; Banegas et al., 2011; US Department of Health and Human Services, 2001).

Ayudar a las personas fumadoras a dejar de fumar es una de las medidas para reducir la morbilidad y la mortalidad relacionada con el tabaquismo (Peto et al., 2000). Los tratamientos para dejar de fumar recomendados por las directrices principales de la práctica clínica han mostrado la misma efectividad en hombres y mujeres (Munafo, Bradburn, Bowes, y David, 2004; Perkins y Scott, 2008). No obstante, las posibles diferencias por sexo halladas en los resultados de dichos tratamientos es un tema controvertido.

Inicialmente, al comienzo de la década de los ochenta, un informe del Director General de Salud Pública (US Department of Health and Human Services, 1980) concluyó que las mujeres tenían mayor dificultad en dejar de fumar, aunque estudios posteriores sugirieron que esta conclusión fue quizás prematura (Whitlock, Vogt, Hollis, y Lichtenstein, 1997). En general, los estudios que evaluaron posibles diferencias en los resultados de tratamientos para dejar de fumar tuvieron resultados diferentes. De esta manera, Whitlock et al. (1997) no halló diferencias por sexo en una intervención clínica breve para dejar de fumar y Croghan et al. (2009) tampoco encontró diferencias en un programa de tratamiento clínico. De la misma manera, un meta análisis de 11 ensayos clínicos con terapia sustitutiva de nicotina (TRN) para dejar de fumar no encontró diferencias por sexo entre fumadores hombres y mujeres (Munafo et al., 2004). Por lo contrario, Osler, Prescott, Godtfredsen, Hein, y Schnohr (1999) encontraron que las mujeres tenían peores resultados al dejar de fumar espontáneamente, mientras que Piper et al. (2010) observaron que las mujeres tenían menor probabilidad de dejar de fumar con éxito cuando se usaba farmacoterapia. Por otro lado, Cepeda, Reynoso, y Erath (2004) observaron que el éxito al dejar de fumar con TSN, estaba mediado por la intensidad del apoyo conductual (mayor intensidad de apoyo para las mujeres) y que las mujeres tenían peores resultados al año, en comparación con los hombres, un resultado similar al de Perkins y Sco- tt (2008). Por último, Scharf y Shiffman (2004) concluyeron que las mujeres tenían menor éxito en dejar de fumar que los hombres, independientemente del tratamiento. En cuanto al seguimiento, muchos estudios han evaluado el éxito de los tratamientos para dejar de fumar por sexo a corto y medio plazo (tres y seis meses de abstinencia), e incluso hasta un año (Croghan et al., 2009; Puente et al., 2011), pero muy pocos han continuado el seguimiento a largo plazo, más allá de los 12 meses (Bjornson et al., 1995; Osler et al., 1999; Wetter et al., 2004), también con resultados contradictorios.

Como se puede observar, las diferencias metodológicas de todos estos estudios pueden explicar, parcialmente, la variedad en los resultados: diferencias en los tratamientos implementados (con o sin farmacoterapia), variedad de los criterios metodológicos para definir abstinencia (autoinformes o medidas bioquímicas), o distintas duraciones de los periodos de seguimiento. Todas estas diferencias dificultan las comparaciones fiables entre estudios.

El objetivo de este estudio fue determinar si existían diferencias entre hombres y mujeres en el éxito de tratamientos para dejar de fumar a corto y largo plazo en un programa de cesación tabáquica que incluye la perspectiva de género.

\section{Métodos}

\section{Participantes}

Se realizó una encuesta telefónica en fumadores atendidos en una Unidad de Tabaquismo entre el 2002 y el 2007 (inclusive). Los participantes fueron personas fumadoras que solicitaron el tratamiento y habían dejado de fumar con éxito al finalizar el mismo. Esta Unidad es un servicio público que ofrece tratamiento a fumadores que lo solicitan para dejar de fumar o que son derivados por su médico de atención primaria (médico de cabecera) o especialista. Los criterios de inclusión para acceder al tratamiento eran ser fumador mayor de 18 años y voluntariamente acordar iniciar el tratamiento, y los criterios de exclusión eran tener un trastorno psiquiátrico no controlado, otra dependencia de drogas activa o, en caso de ser mujer, estar embarazada. Todos los participantes dieron su consentimiento informado por escrito para su inclusión en el estudio.

\section{Intervención}

El programa de cesación tabáquica usa un formato de sesiones grupales de 60 minutos durante 3 meses. Las citas de seguimiento se fijaron de la siguiente manera: primera sesión, el día previo a dejar de fumar; segunda sesión, el día después de dejar de fumar; una cita de refuerzo semanal durante el primer mes; y a los seis, nueve y doce semanas de abstinencia; en resumen, nueve sesiones a lo largo de los tres meses. Todas las sesiones eran en grupo (hombres y 
mujeres juntos) con el mismo día para dejar de fumar fijado para todos.

El tratamiento de cesación tabáquica ofrecido es una intervención que integra varios componentes: tratamiento cognitivo conductual en grupo con tratamiento farmacológico usando las medicaciones recomendadas por las directrices de los tratamientos para dejar de fumar, tales como la Terapia de Reemplazo de Nicotina (TSN), Bupropión y Vareniclina (Fiore et al., 2008); el seguimiento del tratamiento farmacológico se realizó a la vez que las sesiones grupales. Profesionales sanitarios con una experiencia dilatada en terapia grupal dirigieron el tratamiento.

En la terapia cognitiva conductual, todos los participantes recibieron terapia enfocada en prepararse para dejar de fumar, las ventajas de la cesación, cómo afrontar los deseos de fumar y acerca de la prevención de recaídas. La terapia cognitiva también incluyó estrategias específicamente dirigidas a las mujeres para reducir su preocupación por el peso y la imagen corporal, saber cómo romper la relación entre situaciones que dan pie a fumar y el acto de fumar, y estrategias para afrontar emociones negativas.

\section{Medidas}

Durante la cita inicial, y antes de iniciar el tratamiento de cesación tabáquica, se recogieron datos sociodemográficos de todos los participantes (sexo, edad, estado civil, nivel de estudios, situación laboral) y relacionados con fumar, incluyendo el número de cigarrillos fumados por día, años como fumador, número de intentos previos para dejar de fumar $(0,1$ ó 2, y 3 o más), y el grado de dependencia de la nicotina (Test de Fagerström) (Fagerström y Schneider, 1989). El nivel basal de CO se midió usando un cooxímetro Mini Smokerlyzer (Bedfont Scientific Ltd., Rochester, Reino Unido) (Jarvis, Russell, y Saloojee, 1980). Finalmente, se completó un historial médico (hipertensión, niveles de colesterol, enfermedad cardiovascular, hiper- o hipotiroidismo, y cáncer). También informaron sobre su historia previa de ansiedad y/o depresión que requirió tratamiento farmacológico. Dos variables se usaron para recopilar esta información: historia de depresión antes y durante el tratamiento de cesación tabáquica. En esta primera cita, el tratamiento farmacológico se recetó según el perfil individual de cada fumador.

Se recogió la abstinencia continua, es decir, no fumar nada desde el día de dejar de fumar hasta el final del tratamiento (3 meses); valores de CO de $\leq 10$ ppm validaban la consideración de un tratamiento exitoso. Se evaluó el CO en aire espirado en cada cita de seguimiento. Se utilizó el criterio de intención de tratar para evaluar el porcentaje de éxito, la tasa de éxito se definió como la proporción de participantes abstinentes (abstinencia continua y validación del $\mathrm{CO})$ en comparación con la totalidad de participantes que iniciaron el tratamiento. Ambos criterios (éxito y tasa de éxito) se fijaron según las recomendaciones para informar del resultado del tratamiento de cesación tabáquica (Hughes et al., 2003). Se consideraron a todos los participantes que no estuvieron presentes durante la sesión final del tratamiento (semana 12) como fumadores.

\section{Seguimiento}

Con el fin de analizar la abstinencia a largo plazo $(>1$ año), se realizó una encuesta telefónica a todos los participantes abstinentes al final del tratamiento ( 3 meses) entre septiembre de 2008 y junio de 2009. Entrevistadores formados llamaron a cada participante hasta un máximo de cinco veces en dos periodos diferentes de tiempo. Dada la naturaleza telefónica de este seguimiento, se pidió a aquellos participantes que informaron haber dejado de fumar desde que recibió el tratamiento que acudiese a la Unidad para comprobar su abstinencia con medidas bioquímicas.

\section{Análisis estadístico}

Se realizó un análisis descriptivo de la muestra íntegra, con las variables cualitativas expresadas como frecuencias absolutas y la proporción equivalente de cada categoría y las variables cuantitativas como medias y desviaciones estándar. Se compararon las características usando pruebas t para dos muestras para las variables continuas y el test de chi cuadrado para las variables categóricas; se usó el test de chi cuadrado para comparar los resultados a corto plazo. En cualquier caso, se usaron valores $\mathrm{p}$ de $\leq, 05$ a ambos lados para denotar significación estadística.

En el seguimiento telefónico, el tiempo de abstinencia se calculó como el número de meses desde el fin del tratamiento hasta la fecha de dicha entrevista. Se realizó un análisis de supervivencia usando el método Kaplan-Meier para analizar la probabilidad de mantener la abstinencia a largo plazo, con el test de Tarone-Ware para estudiar las posibles diferencias en la supervivencia entre hombres y mujeres (Hughes et al., 2003; Tarone y Ware, 1977). Usamos el test de Tarone-Ware para evaluar los gráficos del estimador de Kaplan-Meier de los diferentes grupos porque dicho test está diseñado para tener una buena potencia en un amplio rango de funciones de supervivencia. Se analizaron los datos usando SPSS $\odot$ versión 15.0.

\section{Resultados}

Un total de 1472 personas, 768 hombres $(52,2 \%)$ y 704 mujeres $(47,8 \%)$, realizaron la historia clínica. La edad media era de 43,2 (DE = 10,3) años. De estos, $170(11,5 \%)$ decidió no participar en el tratamiento de cesación tabáquica, $90(52,9 \%)$ hombres y $80(47,1 \%)$ mujeres. Todas aquellas personas que decidieron no participar en el tratamiento de cesación tabáquica (170) fueron excluidos del estudio y, por lo tanto, no fueron incluidos en los análisis posteriores. 
Tabla 1. Características de los pacientes que iniciaron el tratamiento de cesación tabáquica (2002-2007) $(N=1302)$

\begin{tabular}{|c|c|c|c|c|}
\hline & $\begin{array}{l}\text { Total } \\
\%(\mathrm{~N})\end{array}$ & $\begin{array}{c}\text { Hombres } \\
\%(\mathrm{~N})\end{array}$ & $\begin{array}{l}\text { Mujeres } \\
\%(\mathrm{~N})\end{array}$ & p \\
\hline \multicolumn{5}{|l|}{ Sociodemográficos } \\
\hline Edad (DE) & $43,4(10,2)$ & $44,2(10,4)$ & $42,5(9,9)$ & 002 \\
\hline Estado civil \%(N) & & & & $<, 0001$ \\
\hline Soltero & $23,5(306)$ & $20,1(136)$ & $27,3(170)$ & \\
\hline Divorciado o viudo & $10,0(130)$ & $6,3(43)$ & $13,9(87)$ & \\
\hline Casado & $66,5(866)$ & $73,6(499)$ & $58,8(367)$ & \\
\hline Nivel de estudios \%(N) & & & & $<, 0001$ \\
\hline Básicos & $22,5(293)$ & $25,4(172)$ & $19,4(121)$ & \\
\hline Secundarios & $43,3(564)$ & $47,3(321)$ & $38,9(243)$ & \\
\hline Superiores & $34,2(445)$ & $27,3(185)$ & $41,7(260)$ & \\
\hline Empleo \%(N) & & & & $<, 0001$ \\
\hline Desempleado & $17,1(222)$ & $12,1(82)$ & $22,4(140)$ & \\
\hline Empleado & $82,9(1080)$ & $87,9(596)$ & $77,6(484)$ & \\
\hline \multicolumn{5}{|l|}{ Patrones de consumo } \\
\hline № de cigarrillos/día (DE) & $25,3(10,4)$ & $26,7(11,5)$ & $23,7(8,9)$ & $<, 0001$ \\
\hline Años como fumador (DE) & $26,4(10,1)$ & $27,9(10,7)$ & $24,9(14,8)$ & 003 \\
\hline Intentos previos \%(N) & & & & 019 \\
\hline 0 & $26,5(345)$ & $23,9(162)$ & $29,3(183)$ & \\
\hline $1-2$ & $53(690)$ & $56,6(384)$ & $49,1(306)$ & \\
\hline 3 o más & $20,5(267)$ & $19,5(132)$ & $21,6(135)$ & \\
\hline Test de Fagerström (DE) & $6,2(2,2)$ & $6,3(2,2)$ & $6,2(2,2)$ &, 431 \\
\hline CO basal (DE) & $27,2(15,8)$ & $29,3(16,4)$ & $24,9(14,8)$ &, 003 \\
\hline Medicación con prescripción médica \%(N) & & & & 007 \\
\hline Ninguna & $0,2(2)$ & $0,1(1)$ & $0,2(1)$ & \\
\hline Terapia Sustitutiva de Nicotina & $64,8(844)$ & $69,0(468)$ & $60,3(376)$ & \\
\hline Bupropion & $31,1(405)$ & $27,9(189)$ & $34,6(216)$ & \\
\hline Vareniclina & $3,9(51)$ & $2,9(20)$ & $5,0(31)$ & \\
\hline \multicolumn{5}{|l|}{ Enfermedades \%(N) } \\
\hline Hipertensión & $10,2(133)$ & $12,1(82)$ & $8,2(51)$ & 020 \\
\hline Colesterol & $9,8(127)$ & $12,7(86)$ & $6,6(41)$ & $<, 0001$ \\
\hline Enfermedad cardiovascular & $8,4(109)$ & $11,2(76)$ & $5,3(33)$ & $<, 0001$ \\
\hline Diabetes & $3,5(46)$ & $4,9(33)$ & $2,1(13)$ &, 007 \\
\hline Hipo/Hipertiroidismo & $3,1(41)$ & $0,7(5)$ & $5,8(36)$ & $<, 0001$ \\
\hline Cáncer & $1,5(19)$ & $0,9(6)$ & $2,1(13)$ & ,072 \\
\hline Ansiedad o depresión previa al tratamiento \%(N) & $35,7(465)$ & $24,5(166)$ & 47,9 (299) & $<, 0001$ \\
\hline Ansiedad o depresión durante el tratamiento \%(N) & $10,4(136)$ & $5,9(40)$ & $15,4(96)$ & $<, 0001$ \\
\hline
\end{tabular}


La muestra del estudio incluyó a 1302 personas, de las cuales $678(52,1 \%)$ eran hombres y $624(47,9 \%)$ eran mujeres. La edad media era de 43,4 (DE $=10,2)$ años. En la tabla 1 se muestran las características de la muestra desglosadas por sexo. La edad media de los hombres era mayor que la de las mujeres (44,2 vs. 42,5 años), y era más probable que estuviesen casados $(73,6 \%$ vs. $58,8 \%)$, tuvieran trabajo $(87,9 \%$ vs. $77,6 \%)$, y un nivel de estudios secundarios $(47,3 \%$ vs. $38,9 \%)$, mientras que era más probable que las mujeres hubiesen terminado la universidad (41,7\% vs. $27,3 \%$ de los hombres). En cuanto a las variables relacionadas con fumar, los hombres fumaban más cigarrillos por día que las mujeres (26,7 vs. 23,7), habían sido fumadores durante más tiempo (27,9 vs. 24,9 años), tenían niveles de CO más elevados (29,3 vs. 24,9) y el $56,6 \%$ había intentado dejar de fumar una o dos veces, comparado con el $49,1 \%$ de las mujeres. Todas estas diferencias eran estadísticamente significativas $(\mathrm{p}<, 05)$.

A pesar de las diferencias en sus patrones de consumo, no se hallaron diferencias estadísticamente significativas en la dependencia de la nicotina (6,3 vs. 6,2 puntos; $\mathrm{p}=, 431)$. El análisis de las enfermedades diferentes mostró que era más probable que los hombres tuviesen factores de riesgo de enfermedades cardiovasculares, tales como hipertensión, colesterol y diabetes. Por lo contrario, era más probable que las mujeres tuviesen trastornos psiquiátricos, tales como ansiedad y/o depresión, que requerían tratamiento farmacológico, bien previamente al inicio del estudio o al iniciar su participación en el mismo.

La tasa de éxito a los tres meses de tratamiento, según criterios de intención de tratar, era de 41,3\% (538/1302). No hubo diferencias estadísticamente significativas por sexo, aunque el porcentaje de los que mantuvieron la abstinencia era más elevada para hombres que para mujeres $[43,8 \%$ $(297 / 678)$ vs. $38,6 \%(241 / 624)$ respectivamente; $p=, 058]$.

Un total de 479 de los 538 participantes que completaron el tratamiento con éxito fueron localizados durante el seguimiento telefónico. De los 59 que no contestaron, 24 había cambiado su número de teléfono, 21 quedó ilocalizable en el número estipulado de intentos, ocho no quisieron contestar al cuestionario y seis habían fallecido. Por lo tanto, el seguimiento telefónico se completó con el $89 \%$ (479/538) de los participantes que había terminado el tratamiento con éxito, 47,6\% (256) de los cuales mantuvo la abstinencia desde el día que habían dejado de fumar (el día de cesación); esto representa, por lo tanto, el 19,6\% del total de participantes que había iniciado el tratamiento (256/1302). No hubo diferencias estadísticamente significativas por sexo $(\mathrm{p}=, 519)$. Se comprobó la abstinencia en 191 (53,9\% hombres y 46,1\% mujeres) de los 256 participantes que decían haber dejado de fumar, con valores de CO por debajo de $10 \mathrm{ppm}$; la abstinencia no pudo ser validada en los demás participantes (65), dado que no se presentaron a su cita (Figura 1).
Figura 1. Flujo del estudio

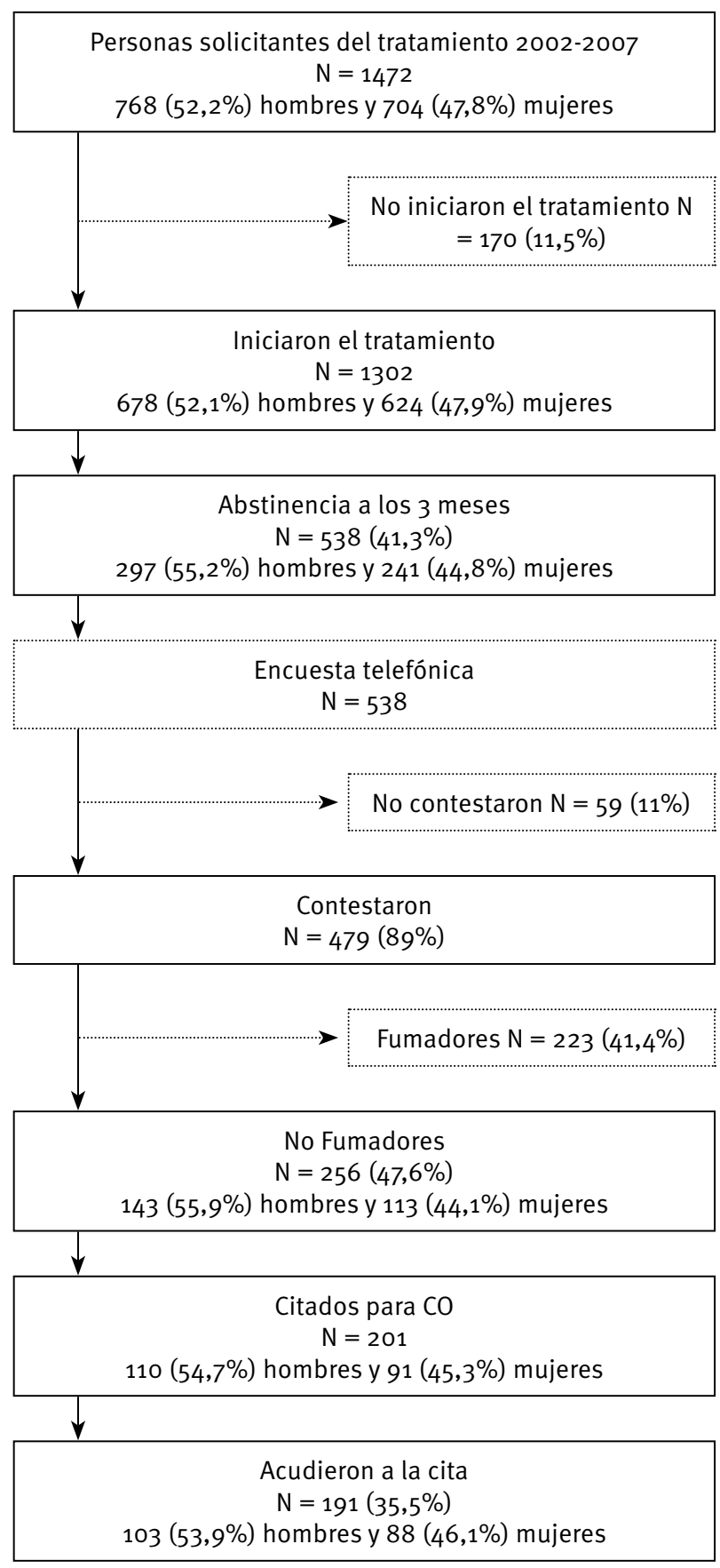

Cuando se comparan las diferencias usando el test de Tarone-Ware, las diferencias detectadas en el análisis de supervivencia usadas para determinar la probabilidad de que los hombres y las mujeres mantuviesen la abstinencia a largo plazo no fueron significativas (Figura 2).

\section{Discusión}

Nuestros resultados muestran que no hubo diferencias de sexo en el éxito a corto y largo plazo del tratamiento de cesación tabáquica que incluye la perspectiva de género, con la misma probabilidad de mantener la abstinencia 
Figura 2. Abstinencia a largo plazo por sexo

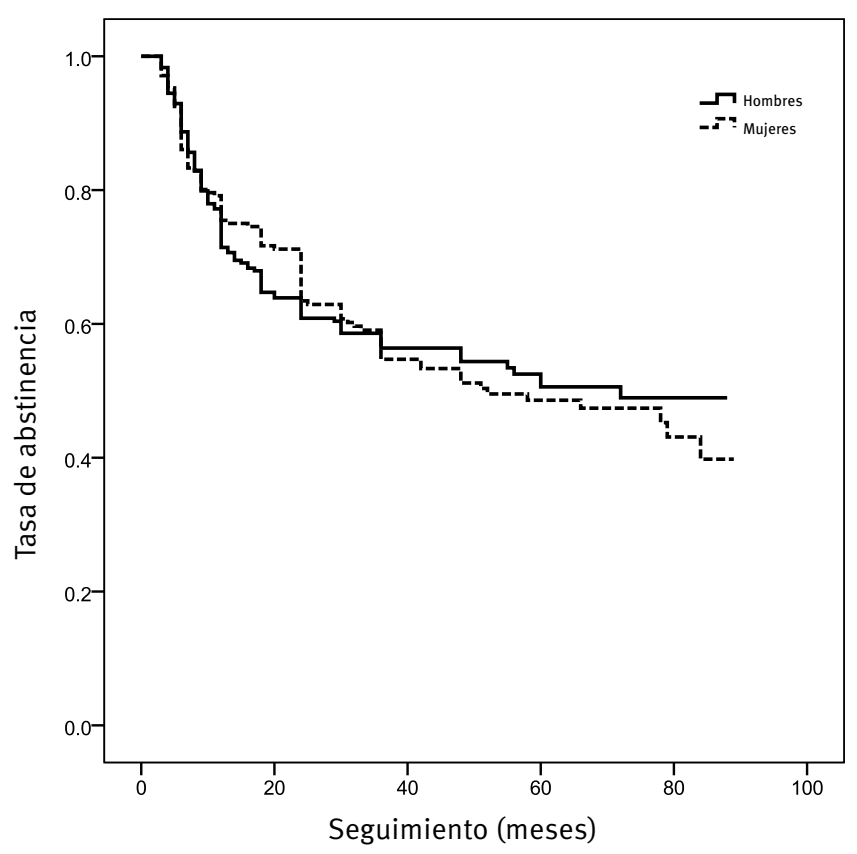

en hombres y mujeres. No obstante, sí encontramos diferencias por sexo en las variables sociodemográficas de las personas que iniciaron el tratamiento en nuestra Unidad. Las mujeres tienden a ser más jóvenes, pero era menos probable, comparado con los hombres, que estuviesen casadas; estas diferencias son similares a las descritas por otros autores (Croghan et al., 2009; Ramon, Bruguera, Fernández, Sanz de Burgoa, y Ramírez, 2009). El porcentaje más alto de hombres con empleo refleja la situación general en España, donde la tasa de empleo masculina es más alta que la femenina. Nuestro estudio también muestra el predominio de mujeres con estudios universitarios en comparación con mayor número de hombres con estudios secundarios; lo mismo fue hallado recientemente por Iliceto, Fino, Pasquariello, D’Angelo Di Paola, y Enea (2013) en Italia. Este aspecto corresponde, para las mujeres, con la fase III del modelo epidemiológico propuesto por López, Collishow y Piha (1994) y la revisión reciente de Thun, Peto, Boreham y Lopez (2012) que incluye a países como España e Italia, en los cuales las mujeres con niveles de estudios superiores tienden a empezar a fumar primero, pero también se deciden a dejar de fumar primero. En cuanto al número elevado de mujeres que solicitaron tratamiento, otros estudios realizados en entornos similares (Unidad de Tabaquismo) también tuvieron números elevados de mujeres, la mayoría con estudios superiores (Croghan et al., 2009; Fernandez et al., 2006; Fidler, Ferguson, Brown, Stapleton y West, 2013).

Según hallazgos previos de nuestro equipo (Marqueta, Nerín, Jiménez-Muro, Gargallo y Beamonte, 2013) y en estudios recientes de otros autores (Chatkin et al., 2006; Iliceto et al., 2013), no se encontraron diferencias estadísticamente significativas entre hombres y mujeres en cuanto al grado de dependencia de la nicotina medida por el Test de Fagerström. Esta "igualdad" refleja el aumento del consumo por parte de las mujeres en los últimos años, contrario a los informes de la literatura de la década de los noventa, que hallaron una dependencia más baja entre las mujeres (Bjornson et al., 1995; Ward, Klesges, Zbikowski, Bliss, y Garvey, 1997). Es más, este estudio se realizó en una Unidad de Tabaquismo especializada, en la cual los hombres y las mujeres que solicitan el tratamiento suelen ser fumadores con una dependencia entre moderada y severa.

Nuestro análisis de las enfermedades detectadas muestra que, según estudios anteriores (Killen, Fortmann, Varady, y Kraemer, 2002; Marqueta, Jiménez-Muro, Beamonte, Gargallo, y Nerín 2010), trastornos de ansiedad y/o depresión son más comunes entre las mujeres, mientras que una proporción mayor de los hombres presenta factores de riesgo de enfermedades cardiovasculares. Ambos aspectos han sido detectados en población general de no fumadores, y pueden ser debidos a diferencias de sexo como resultado de efectos psicosociales y hormonales (Borrell, García-Calvente, y Martí-Boscà, 2004; National Institute of Mental Health, 2009).

En cuanto al éxito del tratamiento de cesación tabáquica, aunque las tasas de éxito eran más altas entre los hombres que entre las mujeres, no encontramos diferencias por sexo a corto plazo como han detectado otros estudios (Croghan et al., 2009; Killen et al., 2002; Puente et al., 2011; Raich et al., 2015; Whitlock et al., 1997) mientras que otros autores, tales como Bohadana, Nilsson, Rasmussen y Martinet (2003), Wetter et al. (2004) y Bjornson et al. (1995) han observado tasas de éxito más altas entre los hombres y una probabilidad más alta de recaída entre las mujeres (Iliceto et al., 2013; Swan, Ward, Carmelli, y Jack, 1993). Las razones dadas para justificar el peor resultado de los tratamientos de cesación tabáquica entre mujeres sugieren que las mujeres perciben el acto de fumar como una estrategia para reducir efectos negativos (el estrés, por ejemplo) y/o para aumentar los positivos (Xu et al., 2008). Es ampliamente conocido que las mujeres fuman por motivos diferentes a los de los hombres, por ejemplo, para reducir estados negativos (tristeza, ansiedad, etc.), y que sus preocupaciones son diferentes a la hora de dejar de fumar, incluyendo el control de su peso y la aparición de síntomas depresivos (Croghan et al., 2009; US Department of Health and Human Services, 2001; WHO, 2001). Por lo tanto, se ha sugerido que la conducta de fumar entre las mujeres puede estar más influenciada por componentes conductuales y menos por la dependencia de la nicotina que los hombres y, por tanto, el tratamiento debe ser personalizado para mujeres con el fin de aumentar sus probabilidades de mantener la abstinencia (Bohadana et al., 2003). Algunos estudios han observado que el resultado de las mujeres en un programa de cesación tabáquica está influenciado por la intensidad del apoyo conductual, con un apoyo mayor para mujeres, pero sin incluir recomendaciones específicas para mujeres (Cepeda et al., 
2004). En nuestro estudio, para evitar recaídas, incluimos estrategias específicas de las mujeres tales como factores asociados al peso, el afrontamiento de situaciones negativas y la gestión del estrés, lo que puede explicar la ausencia de diferencias entre hombres y mujeres en cuanto a los resultados del tratamiento de cesación tabáquica.

Es más, Croghan et al. (2009), ajustando por las características basales de los fumadores, observaron que la probabilidad de abstinencia no se diferenciaba por sexo y sugirieron que otras características podrían explicar las diferencias observadas en cuanto a los resultados de abstinencia de tabaco entre fumadores hombres y mujeres (i.e., tasa basal de fumar, historia de depresión, etc.), que varían por sexo. De la misma manera, nuestro grupo, usando una metodología similar, no halló diferencias por sexo en el resultado de los programas de cesación tabáquica, lo que sugiere que los predictores de éxito en la abstinencia son diferentes entre los hombres y las mujeres (Marqueta et al., 2013). En otras palabras, y como han sugerido otros autores anteriormente, la tasa de éxito de programas de cesación tabáquica es similar para ambos sexos, pero el proceso es diferente para hombres y mujeres (Whitlock et al., 1997). Estos resultados subrayan la importancia de individualizar el tratamiento para fumadores en función del sexo.

En nuestro estudio, el éxito a largo plazo se puede ver en la Figura 1, y, acorde con los resultados de Chatkin et al. (2006), los hombres y las mujeres tienen la misma probabilidad de mantener la abstinencia a largo plazo. Conociendo los resultados a largo plazo, merece la pena destacar que los hombres y las mujeres tienen el mismo éxito después de participar en un programa de cesación tabáquica que incluye la perspectiva de género, congruente con los resultados hallados a corto plazo.

Entre las limitaciones de nuestro estudio, hemos de destacar que la población objeto del estudio no era representativa de la población fumadora general, dado que sólo incluía a fumadores que solicitaron tratamiento en una Unidad de Tabaquismo especializada. A pesar de esto, la muestra de fumadores es suficientemente grande para permitir el análisis de las diferencias entre hombres y mujeres en cuanto al éxito del tratamiento y, por lo tanto, es adecuado para los fines propuestos. Además, los estudios realizados en Unidades de Tabaquismo suelen analizar la totalidad de los pacientes tratados y no usan muestras (Fernandez et al., 2006). Otra limitación de nuestro estudio podría ser el número de pacientes que informaron durante la encuesta telefónica que no fumaban y que no participaron en la verificación bioquímica (Figura 1). No obstante, esta situación es muy frecuente en los estudios que evalúan la abstinencia a largo plazo, en los cuales estos pacientes son considerados fumadores (Álvarez et al., 2015); nuestro estudio aplicó el mismo criterio.

Por otro lado, una de las fortalezas de nuestro estudio es el tamaño y naturaleza clínica de la muestra y el seguimiento a largo plazo, a diferencia de otros estudios que suelen ser ensayos clínicos con periodos de seguimiento más breves. Es más, usamos la abstinencia continua, y esta medida es la más rigurosa, considerada por muchos como la medida de referencia, dado que requiere de un periodo más largo de abstinencia que otras medidas y es, por lo tanto, más probable que represente la abstinencia a largo plazo; además, validamos la abstinencia con CO. Así mismo, siguiendo los criterios de intención de tratar aplicados para evaluar el porcentaje de éxito, todos los participantes que no completaron dicha validación fueron considerados fumadores. De forma similar, y según las recomendaciones de SRNT (2002) (Hughes et al., 2003), se realizó un análisis de supervivencia usando el método Kaplan-Meier para analizar la probabilidad de mantener la abstinencia a largo plazo. Este método aporta información más detallada que la de un punto de corte sencillo como tasa, ya que refleja la evolución temporal e informa sobre probabilidad, y por lo tanto refleja la situación actual del paciente con mayor precisión. Dado que fumar no es un proceso estático en el tiempo (Prochaska y DiClemente, 1983), parece más adecuado el uso de técnicas dinámicas, tales como el análisis de supervivencia, para valorar dichos resultados. Por lo contrario, muchos estudios evalúan la abstinencia únicamente mediante el autoinforme para fijar el punto de corte de una muestra.

En resumen, nuestro estudio muestra que no hay diferencias por sexo en cuanto al resultado del tratamiento de cesación tabáquica cuando se sigue un tratamiento recomendado por las guías de práctica clínica. Estas recomendaciones incluyen la personalización del tratamiento según las características de cada fumador. Esto significa que es necesario adaptar el tratamiento de cesación tabáquica teniendo en cuenta las diferentes preocupaciones y necesidades de mujeres y hombres.

\section{Reconocimientos}

Este estudio ha sido financiado por el Grupo de Investigación en Tabaquismo B86, del Gobierno de Aragón, (España) y el Ministerio de Ciencia e Innovación. Subprograma de Acciones Complementarias (Ref: PSI2008-05177-E) (España).

\section{Conflicto de intereses}

Los autores declaran la inexistencia de conflictos de intereses.

\section{Referencias}

Álvarez, F. J., Ferrer, M., Ruiz, A., Medina, J. F., Romero, B., Sáez, A., y Romero, A. (2015). Predictors of 10-year smoking abstinence in smokers abstinent for 1 year after treatment. Addiction, 111, 545-551. doi: 10.1111/add.13220

Amos, A., Greaves, L., Nichter, M., y Bloch, M. (2012). Women and tobacco: a call for including gender in tobacco 
control research, policy and practice. Tobacco Control, 21, 236-243. doi: 10.1136/tobaccocontrol-2011-050280.

Banegas, J. R., Díez-Gañán, L., Bañuelos-Marco, B., González-Enríquez, J., Villar-Álvarez, F., Martín-Moreno, J. M.,... Jiménez-Ruiz, C. (2011). Smoking-attributable deaths in Spain. Medicina Clínica, 136, 97-102. doi: 10.1016/j.medcli.2010.03.039.

Bjornson, W., Rand, C., Connett, J. E., Lindgren, P., Nides, M., Pope, F., ...O’Hara, P. (1995). Gender differences in Smoking Cessation after 3 Years in the Lung Health Study. American Journal of Public Health, 85, 223-230.

Bohadana, A., Nilsson, F., Rasmussen, T., y Martinet, Y. (2003). Gender differences in quit rates following smoking cessation with combination nicotine therapy: Influence of baseline smoking behavior. Nicotine $\mathcal{E}$ Tobacco Research, 5, 111-116.

Borrell, C., García-Calvente, M. M., y Martí-Boscà, J. V. (2004). La salud pública desde la perspectiva de género y clase social. Gaceta Sanitaria, 18, 2-6.

Chatkin, J. M., Abreu, C. M., Blanco, D. C., Tonietto, R., Scaglia, N., Wagner, M. B., y Fritscher, C. C. (2006). No gender difference in effectiveness of smoking cessation treatment in a Brazilian real-life setting. The International Journal of Tuberculosis and Lung Disease, 10, 499-503.

Cepeda, A., Reynoso, J. T., y Erath, S. (2004). Meta-Analysis of the Efficacy of Nicotine Replacement Therapy for Smoking Cessation: Differences Between Men and Women. Journal of Consulting and Clinical Psychology, 72, 712-722.

Croghan, I. T., Ebbert, J. O., Hurt, R. D., Hays, J. T., Dale, L. C. Warner, N. y Schroeder, D. R. (2009). Gender differences among smokers receiving interventions for tobacco dependence in a medical setting. Addictive Behaviors, 34, 61-67. doi: 10.1016/j.addbeh.2008.08.010.

Fagerström, K. O. y Schneider, N. G. (1989). Measuring nicotine dependence: a review of the Fagerström Tolerance Questionnaire. Journal of Behavioral Medicine, 12, 159-182.

Fernandez, E., Schiaffino, A., Borrell, C., Benach, J., Ariza, C., Ramon, J. M.,... Kunst, A. (2006). Social class, education, and smoking cessation: Long-term follow-up of patients treated at a smoking cessation unit. Nicotine $\mathcal{E}^{\circ}$ Tobacco Research, 8, 29-36.

Fidler, J., Ferguson, S. G., Brown, J., Stapleton, J., y West, R. (2013). How does rate of smoking cessation vary by age, gender and social grade? Findings from a population survey in England. Addiction, 108, 1680-1685. doi: 10.1111/add.12241.

Fiore, M. C., Jaen, C. R., Baker, T. B., Baikey, W. C., Benowitz, N. L., Curry, S. J., ... Wewers, M. E. (2008). Treating tobacco use and dependence. A report of the US Surgeon General. Rockville, MD: U.S. Department of Health and Human Services, Public Health Service, 257p.

Hughes, J. R., Keely, J. P., Niaura, R. S., Ossip-Klein, D. J., Richmond, R. L, y Swan, G. E. (2003). Measures of abs- tinence in clinical trials: issues and recommendations. Nicotine Eo Tobacco Research, 5, 13-25.

Iliceto, P., Fino, E., Pasquariello, S., D’Angelo Di Paola, M. E., y Enea, D. (2013). Predictors of success in smoking cessation among Italian adults motivated to quit. Journal of Substance Abuse Treatment, 44, 534-540. doi: 10.1016/j. jsat.2012.12.004.

Jarvis, M. J., Russell, M. A., y Saloojee, Y. (1980). Expired air carbon monoxide: a simple breath test of tobacco smoke intake. British Medical Journal, 281, 484-485.

Killen, J. D., Fortmann, S. P., Varady, A., y Kraemer, H. C. (2002). Do Men Outperform Women in Smoking Cessation Trials? Maybe, But Not by Much. Experimental and Clinical Psychopharmacology, 10, 295-301.

López, A. D., Collishow, H. E., y Piha, T. (1994). A descriptive model of the cigarette epidemic in developed countries. Tobacco Control, 3, 242-247.

López, A. D., Mathers, C. D., Ezzati, M., Jamison, D. T., y Murray, C. J. (2006). Global and regional burden of disease and risk factors, 2001: systematic analysis of population health data. Lancet, 367, 1747-1757.

Marqueta, A., Nerín, I., Jiménez-Muro, A., Gargallo, P., y Beamonte, A. (2013). Factores predictores de éxito según género en el tratamiento del tabaquismo. Gaceta Sanitaria, 27, 26-31. doi: 10.1016/j.gaceta.2011.12.011

Marqueta, A., Jiménez-Muro, A., Beamonte, A., Gargallo, P., y Nerín, I. (2010). Evolución de la ansiedad en el proceso de dejar de fumar en fumadores que acuden a una Unidad de Tabaquismo. Adicciones, 22, 317-324.

Munafo, M., Bradburn, M., Bowes, L., y David, S. (2004). Are there sex differences in transdermal nicotine replacement therapy patch efficacy? A meta-analysis. Nicotine Eo Tobacco Research, 6, 769-776.

National Institute of Mental Health, 2009. Women and depression. Recuperado de: http://www.nimh.nih.gov/health/ publications/women-and-depression-discovering-hope/ depression-what-every-woman-should-know.pdf

Osler, M., Prescott, E., Godtfredsen, N., Hein, H. O., y Schnohr, P. (1999). Gender and determinants of smoking cessation: a longitudinal study. Preventive Medicine, 29, 57-62.

Perkins, K. A. y Scott, J. (2008). Sex differences in longterm smoking cessation rates due to nicotine patch. Nicotine $\mathcal{E}$ Tobacco Research, 10, 1245-1251. doi: 10.1080/14622200802097506.

Peto, R., Darby, S., Deo, H., Silcocks, P., Whitley, E., y Doll, R. (2000). Smoking, smoking cessation, and lung cancer in the UK since 1950: combination of national statistics with two case-control studies. British Medical Journal, 321, 323-329.

Piper, M. E., Cook, J. W., Schlam, T. R., Jorenby, D. E., Smith, S. S., Bolt, D. M., y Loh, WY. (2010). Gender, race, and education differences in abstinence rates among participants in two randomized smoking cessation trials. 
Nicotine $\mathcal{E}$ Tobacco Research, 12, 647-657. doi: 10.1093/ ntr/ntq067.

Prochaska, J. O. y DiClemente, C. C. (1983). Stages and processes of self change of smoking: toward an integrative model of change. Journal of Consulting and Clinical Psychology, 51, 390-395.

Puente, D., Cabezas, C., Rodriguez-Blanco, T., Fernández-Alonso, C., Cebrian, T., Torrecilla, M.,... Martín, C. (2011). The role of gender in a smoking cessation intervention: a cluster randomized clinical trial. BMC Public Health, 11, 369. doi: 10.1186/1471-2458-11-369.

Raich, A., Martínez-Sánchez, J. M., Marquilles, E., Rubio, L., Fu, M., y Fernández, E. (2015). Smoking cessation after 12 months with multi-component therapy. Adicciones, 27, 37-46.

Ramon, J. M., Bruguera, E., Fernández, C., Sanz de Burgoa, V., y Ramírez, E. (2009). Motivos para dejar de fumar en España en función del sexo y la edad. Gaceta Sanitaria, 23, 539.e1-539.e6. doi: 10.1016/j.gaceta.2009.07.004.

Scharf, D. y Shiffman, S. (2004). Are there gender differences in smoking cessation, with and without bupropion? Pooled-and meta-analyses of clinical trials of Bupropion SR. Addiction, 99, 1462-1469.

SRNT Subcommittee on Biochemical Verification. (2002). Biochemical verification of tobacco use and cessation. Nicotine E Tobacco Research, 4, 149-159.

Swan, G. E., Ward, M. M., Carmelli, D., y Jack, L. M. (1993). Differential rates of relapse in subgroups of male and female smokers. Journal of Clinical Epidemiology, 46, 10411053.

Tarone, R. E. y Ware, J. (1977). On distribution-free tests for equality of survival distributions. Biometrika 64, 156-160.

Thun, M., Peto, R., Boreham, J., y Lopez, A. D. (2012). Stages of the cigarette epidemic on entering its second century. Tobacco Control, 21, 96-101. doi: 10.1136/tobaccocontrol-2011-050294.

US Department of Health and Human Services (USDHHS) (2001). Women and Smoking: A report of the Surgeon General. Rockville, MD. Public Health Service, Office of the Surgeon General.

US Department of Health and Human Services (USDHHS) (1980). The health consequences of smoking for women. A report of the Surgeon General. Rockville, MD. Public Health Service, Office of the Surgeon General.

Ward, K., Klesges, R. C, Zbikowski, S. M., Bliss, R. E., y Garvey, A. J. (1997). Gender differences in the outcome of a unaided smoking cessation attempt. Addictive Behaviors, 22, 521-533.

Wetter, D. W., Cofta-Gunn, L., Fouladi, R. T., Cinciripini, P. M, Sui, D., y Gritz, E. (2004). Late relapse/sustained abstinence among former smokers: a longitudinal study. Preventive Medicine, 39, 1156-1163.

Whitlock, E. P., Vogt, M. T., Hollis, J. F., y Lichtenstein, E. (1997). Does gender affect response to a brief clinic-ba- sed smoking intervention? American Journal of Preventive Medicine, 13, 159-166.

World Health Organization. Women and the tobacco epidemic. Challenges for the 21st century. Geneva: WHO, 2001. Retrieved from: http://whqlibdoc.who.int/hq/ 2001/WHO_NMH_TFI_01.1.pdf

Xu, J., Azizian, A., Monterosso, J., Domier, C. P., Brody, A. L., Fong, T. W., y London, E. D. (2008). Gender effects on mood and cigarette craving during early abstinence and resumption of smoking. Nicotine $\mathcal{E}$ Tobacco Research, 10, 1653-1661. doi: 10.1080/14622200802412929. 